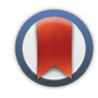

CrossMark

Cite this: Food Funct., 2016, 7, 2909

DOI: $10.1039 / c 6 f o 90021 f$

www.rsc.org/foodfunction

\title{
Correction: Microencapsulation of probiotics in hydrogel particles: enhancing Lactococcus lactis subsp. cremoris LM0230 viability using calcium alginate beads
}

\author{
Timothy W. Yeung, ${ }^{a}$ Izlia J. Arroyo-Maya, ${ }^{a}$ David J. McClements ${ }^{a, b}$ and \\ David A. Sela*a,c,d \\ Correction for 'Microencapsulation of probiotics in hydrogel particles: enhancing Lactococcus lactis \\ subsp. cremoris LM0230 viability using calcium alginate beads' by Timothy W. Yeung et al., Food Funct., \\ 2016, 7, 1797-1804
}

In section 2.1 of this article, 'Bacteria propagation and general growth conditions', it is erroneously reported that Lactococcus lactis subsp. cremoris LM0230 were routinely propagated at $30^{\circ} \mathrm{C}$. The correct temperature is $37^{\circ} \mathrm{C}$.

The Royal Society of Chemistry apologises for these errors and any consequent inconvenience to authors and readers.

\footnotetext{
${ }^{a}$ Department of Food Science, University of Massachusetts, Amherst, MA 01003, USA. E-mail: davidsela@umass.edu; Tel: +14135451010

${ }^{b}$ Department of Biochemistry, Faculty of Science, King Abdulaziz University, P. O. Box 80203, Jeddah 21589, Saudi Arabia

${ }^{c}$ Center for Bioactive Delivery, Institute of Applied Life Science, University of Massachusetts, Amherst, MA 01003, USA

${ }^{d}$ Center for Microbiome Research, University of Massachusetts Medical School, Worcester, MA 01655, USA
} 